\title{
MCt Avalanche Photodiode Detector For TWo-Micron ACTIVE REMOTE SENSING APPLICATIONS
}

\author{
Tamer F. Refaat, Upendra N. Singh, Mulugeta Petros and Ruben Remus \\ NASA Langley Research Center \\ Hampton, Virginia, USA \\ tamer.f.refaat@nasa.gov
}

\begin{abstract}
Mercury Cadmium Telluride electron initiated avalanche photodiodes demonstrated a breakthrough in lidar active remote sensing technology. A lidar detection system, based on an array of these devices, was integrated and characterized for 2- $\mu \mathrm{m}$ applications. Characterization experiments were focused on evaluating the dark current, gain and responsivity variations with bias voltage. Quantum efficiency and input dynamic range including noise-equivalent-power and maximum detectable power, were calculated from these results. Operating the detection system using four pixels at $77.6 \mathrm{~K}, 12 \mathrm{~V}$ bias resulted in a current responsivity of $615.8 \mathrm{~A} / \mathrm{W}$ and a voltage responsivity of 1.45 GV/W. Minimum detectable power of $14 \mathrm{pW}$ was obtained, which is equivalent to $5.7 \mathrm{fW} / \mathrm{Hz}^{1 / 2}$ noise-equivalent-power, indicating an average noise-equivalent-power of $1.4 \mathrm{fW} / \mathrm{Hz}^{1 / 2}$ per pixel. Work is in progress to integrate and validate this detection system using a newly developed triple-pulse integrated path differential absorption lidar for simultaneous and independent atmospheric measurements of water vapor and carbon dioxide.
\end{abstract}

Keywords -IR detector; MCT; lidar; DIAL; IPDA

\section{INTRODUCTION}

Infrared (IR) detectors are required for several active remote sensing applications using the lidar technique. While these detectors are commercially available, specific requirements of lidar instruments constrain the choices of devices and vendors. These requirements cover both the science objectives and the operating conditions that are tailored for each instrument. A detector in a lidar instrument could be subjected to temperature fluctuations, high vibrations and different background radiation and still have to perform without compromising sustained detection quality, sensitivity and reliability. For example, IR detectors for airborne nadir lidar operation are subjected to high power magnitude due to high reflectivity clouds and Earth surface returns. The same detectors for zenith lidar operation are constrained by low power returns that limit noise specifications. To meet such requirements, each detector is selected with specific parameters that may result in increased manufacturing cost and time.

IR detectors based on Mercury Cadmium Telluride (HgCdTe or MCT) are attractive for space-based lidar applications. Compared to other detector materials MCT is a mature and reliable technology amenable to space qualification. MCT is an alloy semiconductor exhibiting tunable bandgap energy that covers IR quantum detector applications in the short wavelength (SWIR: 1-3 $\mu \mathrm{m}$ ), middle wavelength (MWIR: 3-5 $\mu \mathrm{m}$ ), long wavelength (LWIR: 8-14 $\mu \mathrm{m}$ ), and very long wavelength (VLWIR: 14-30 $\mu \mathrm{m}$ ) IR ranges [1]. Operating at $77 \mathrm{~K}$, MCT sensitivity in LWIR and VLWIR is superior than thermal detectors and comparable in performance with other quantum detectors that require cooling to $4 \mathrm{~K}$ [2]. Low dielectric constant of MCT provides low junction capacitance for high frequency and high bandwidth applications. These detectors are commercially available in both photoconductive and photovoltaic configurations with single element and multipixel focal plane array (FPA) formats.

Recent development of advanced single-charge-carrier MCT electron-initiated avalanche photodiodes (e-APD) indicate a breakthrough in lidar detection technology [3]. These devices were validated for airborne lidar operation at 1.6- $\mu \mathrm{m}$ at NASA Goddard Space Flight Center (GSFC) using the integrated path differential absorption (IPDA) lidar technique [4]. The latest MCT e-APD arrays are manufactured in $4 \times 4$ pixel-format with $80 \times 80 \mu \mathrm{m}^{2}$ pixel-size. They exhibit IR sensitivity from $1-\mu \mathrm{m}$ up to $4-\mu \mathrm{m}$. In coordination through NASA's Earth Science Technology Office (ESTO), GSFC integrated a state-of-the-art lidar detection system using this newly developed e-APD. This direct detection system was optimized for 2- $\mu \mathrm{m}$ pulsed lidar development at NASA Langley Research Center (LaRC) [5]. Plans are focused to integrate this detection system into the newly developed triplepulse IPDA lidar for simultaneous and independent atmospheric measurements of water vapor and carbon dioxide and future differential absorption lidar (DIAL) applications [67]. The characterization results of this MCT e-APD lidar detection system at $2-\mu \mathrm{m}$ are presented in this paper to determine the instrument dynamic range.

\section{MCT E-APD DETECTION SYSTEM}

The MCT e-APD consists of 16 pixels arranged in $4 \times 4$ pixel-array with readout electronics that enable access to each pixel through an individual, variable gain, trans-impedance amplifier (TIA), as shown in Fig. 1 [4]. An output summing amplifier (SUM) produces the summation of a specific number of pixels as selected by the operator. This unit includes a buffer amplifier with $7.28 \mathrm{~V} / \mathrm{V}$ gain. The TIA and SUM amplifiers are coupled through either dc or ac coupling using hardware connectors. The detection system SUM output is limited to 1.4 $\mathrm{V}$ and terminated by the $50 \Omega$ input impedance of an advanced, 12-bit, $1 \mathrm{GS} / \mathrm{s}$ digitizer. The detection system bandwidth is set 


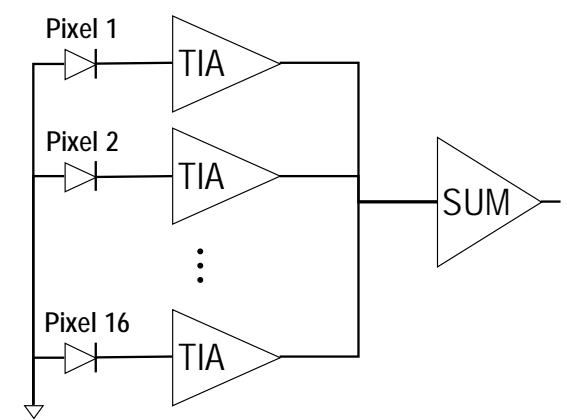

Fig 1. Schematic of the MCT e-APD $4 \times 4$ pixel array with readout electronics that enable access to each pixel through an individual, variable gain TIA with the SUM amplifier.
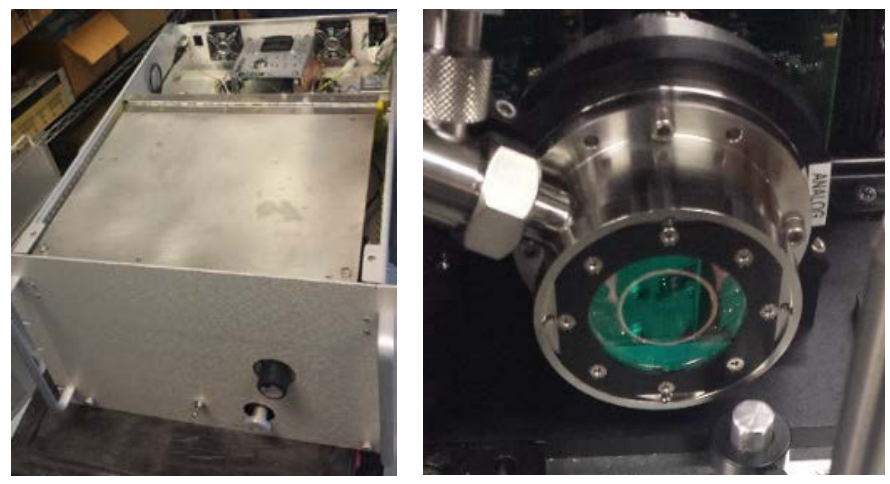

Fig 2. MCT e-APD detection system rack mountable chassis (left) that houses the detector chamber (right) as well as cryo-cooling system, vacuum circuit and electronics.

to $6 \mathrm{MHz}$. The detector is integrated inside a vacuum chamber, which allows cooling the device with a cryo-cooler, down to 77 $\mathrm{K}$, to reduce dark current and noise. The e-APD and readout electronics are integrated inside a rack mountable chassis, shown in Fig. 2, which includes the cooling system and the vacuum circuit for thermal isolation. Additional custom designed aft-optics couples the input radiation, using a $300 \mu \mathrm{m}$ diameter optical fiber, and focuses it onto the center $2 \times 2$ pixels. Reducing the number of operating pixels results in reducing the total noise of the detection system. The detection system includes an optical narrow band-pass cold filter, centered at 2$\mu \mathrm{m}$, to enhance the device response by background reduction. In addition, a protection circuit was implemented to shut down the detection system in response to extreme optical power input to avoid device damage. Operational software allows activating a selected number of pixels, changing the bias voltage and the TIA gains of the selected pixels as well as monitoring different aspects of the system such as vacuum pressure and operating temperature.

\section{DETECTION SYSTEM CHARACTERIZATION}

The objective of the detection system characterization is to project the performance of the MCT e-APD for 2- $\mu \mathrm{m}$ lidar applications. Characterization experiments were focused on evaluating the whole detection system, including the digitizer, while activating only the $2 \times 2$ center pixels. Experiments were conducted to obtain dark current, gain and responsivity variations with bias voltage at different TIA gain settings. The characterization results were used to obtain the quantum efficiency and input dynamic range including the noiseequivalent-power (NEP) and maximum detectable power. Each data record consists of the statistical results of $30 \mathrm{M}$ samples obtained at steady state conditions. A $2-\mu \mathrm{m}$ semiconductor fiber coupled laser source was used in conjunction with optical fiber attenuators to produce a continuous input radiation to the detection system. An optical spectrum analyzer scan of the source radiation indicated $2051.04 \mathrm{~nm}$ centered wavelength and $6 \mu \mathrm{W}$ output optical power, as shown in Fig. 3.

Table I lists the measurement results of the detection system current and voltage responsivities and the corresponding quantum efficiencies obtained at different TIA gain settings at $0 \mathrm{~V}$ bias and $77.6 \mathrm{~K}$. Measurements were obtained while attenuating the radiation source power to $296.75 \mathrm{nW}$. The current responsivity, $\mathfrak{R}_{\mathrm{i}}(\mathrm{A} / \mathrm{W})$, and voltage responsivity, $\mathfrak{R}_{\mathrm{v}}$ $(\mathrm{V} / \mathrm{W})$ are referred to the detection system input and digitizer output, respectively. The current responsivity is given by

$\Re_{i}=\frac{\left(I_{s}-I_{d}\right)}{P}=\frac{q}{h \cdot c} \cdot \lambda \cdot \eta \cdot G$

where $I_{d}$ and $I_{s}$ are the dark and total signal currents, $P$ is the input radiation power, $\mathrm{q}$ is the electron charge, $\mathrm{h}$ is the Plank's constant, $\mathrm{c}$ is the speed of light, $\lambda$ is the wavelength, and $\eta$ and $\mathrm{G}$ are the e-APD quantum efficiency and avalanche multiplication gain, respectively. The voltage responsivity is given by

$$
\Re_{v}=\frac{\left(V_{s}-V_{d}\right)}{P}=R_{f} \cdot A \cdot \Re_{i}
$$

where $V_{d}$ and $V_{s}$ are the dark and total signal voltages, $R_{f}$ is the TIA feedback resistance and $A$ is the buffer amplifier gain.

Table 1. MCT e-APD detection System characterization results at $0 \mathrm{~V}$ bias, 77.6 $\mathrm{K}$ temperature and $2051.04 \mathrm{~nm}$ radiation wavelength.

\begin{tabular}{|l|c|c|c|c|c|c|c|}
\hline TIA Gain & 0 & 1 & 2 & 3 & 4 & 5 & 6 \\
\hline $\mathrm{R}_{\mathrm{f}}(\mathrm{k} \Omega)$ & 46.29 & 53.98 & 64.98 & 81.49 & 107.26 & 161.36 & 321.7 \\
\hline $\mathrm{V}_{\mathrm{d}}(\mathrm{mV})$ & 168.4 & 168.0 & 168.7 & 168.1 & 169.2 & 167.9 & 169.8 \\
\hline $\mathrm{V}_{\mathrm{s}}(\mathrm{mV})$ & 274.7 & 290.2 & 316.9 & 354.0 & 409.1 & 529.9 & 898.1 \\
\hline $\mathrm{V}_{\mathrm{s}}-\mathrm{V}_{\mathrm{d}}(\mathrm{mV})$ & 106.3 & 122.2 & 148.2 & 185.9 & 239.9 & 361.9 & 722.3 \\
\hline $\mathrm{I}_{\mathrm{s}}-\mathrm{I}_{\mathrm{d}}(\mathrm{nA})$ & 315 & 311 & 313 & 313 & 307 & 308 & 309 \\
\hline$\Re_{\mathrm{i}}(\mathrm{A} / \mathrm{W})$ & 1.063 & 1.048 & 1.056 & 1.056 & 1.035 & 1.038 & 1.041 \\
\hline$\Re_{\mathrm{v}}(\mathrm{MV} / \mathrm{W})$ & 0.358 & 0.412 & 0.499 & 0.626 & 0.808 & 1.219 & 2.437 \\
\hline$\eta(\%)$ & 64.26 & 63.34 & 63.82 & 63.83 & 62.58 & 62.77 & 62.91 \\
\hline
\end{tabular}

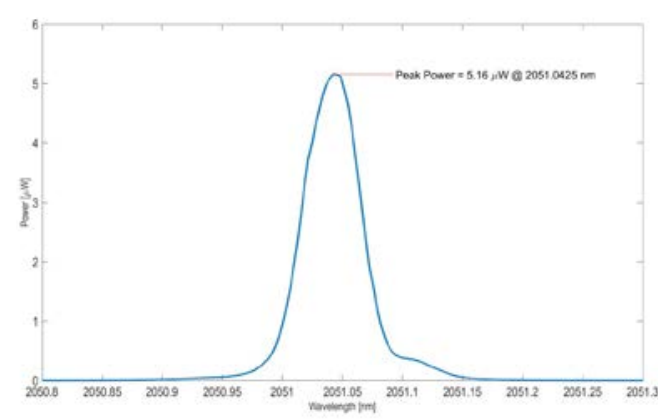

Fig 3. Optical spectrum analyzer scan of the 2- $\mu \mathrm{m}$ semiconductor fiber coupled laser source used for MCT e-APD detection system characterization. Source radiation is centered at $2051.04 \mathrm{~nm}$ with peak output optical power of $5.2 \mu \mathrm{W}$. Fiber attenuators were used to reduce the power to avoid detector saturation. 
Eq. 1 sets the maximum limit of the current responsivity to 1.65 A/W assuming $100 \%$ quantum efficiency. Although current responsivities and quantum efficiencies should be independent of the TIA gain setting, fluctuations observed in the results of Table I are due to uncertainties in TIA gain determination. At $2051.04 \mathrm{~nm}$ wavelength, using $0 \mathrm{~V}$ and $77.6 \mathrm{~K}$ operating bias and temperature, results indicated $1.048 \mathrm{~A} / \mathrm{W}$ mean current responsivity corresponding to a quantum efficiency of $63.36 \%$.

Fig. 4 shows the detection system output voltage variation with the applied bias voltage obtained in dark conditions. These results correspond to the e-APD dark current amplified using different TIA gain settings. For each setting, dark output voltage and voltage noise were obtained as the mean and standard deviation for the $30 \mathrm{M}$ sample records. Up to $6 \mathrm{~V}$, dark measurements exhibit a constant value dominated by the detection system electronics. Above $6 \mathrm{~V}$ bias rapid increase in dark measurements result due to the increase in the e-APD gain. Appling the $296.75 \mathrm{nW}$ radiation, at $2051.04 \mathrm{~nm}$, to the detection system through fiber coupling, voltage responsivity was obtained using Eq. 2. Fig. 5 shows the variation of the voltage responsivity with the applied bias for the different TIA gains. Fig. 6 shows the e-APD avalanche multiplication gain obtained using Eqs. 1 and 2. This gain starts to increase at about $2 \mathrm{~V}$ and reaches a maximum of 587.5 at $12 \mathrm{~V}$, independent of the TIA feedback setting.

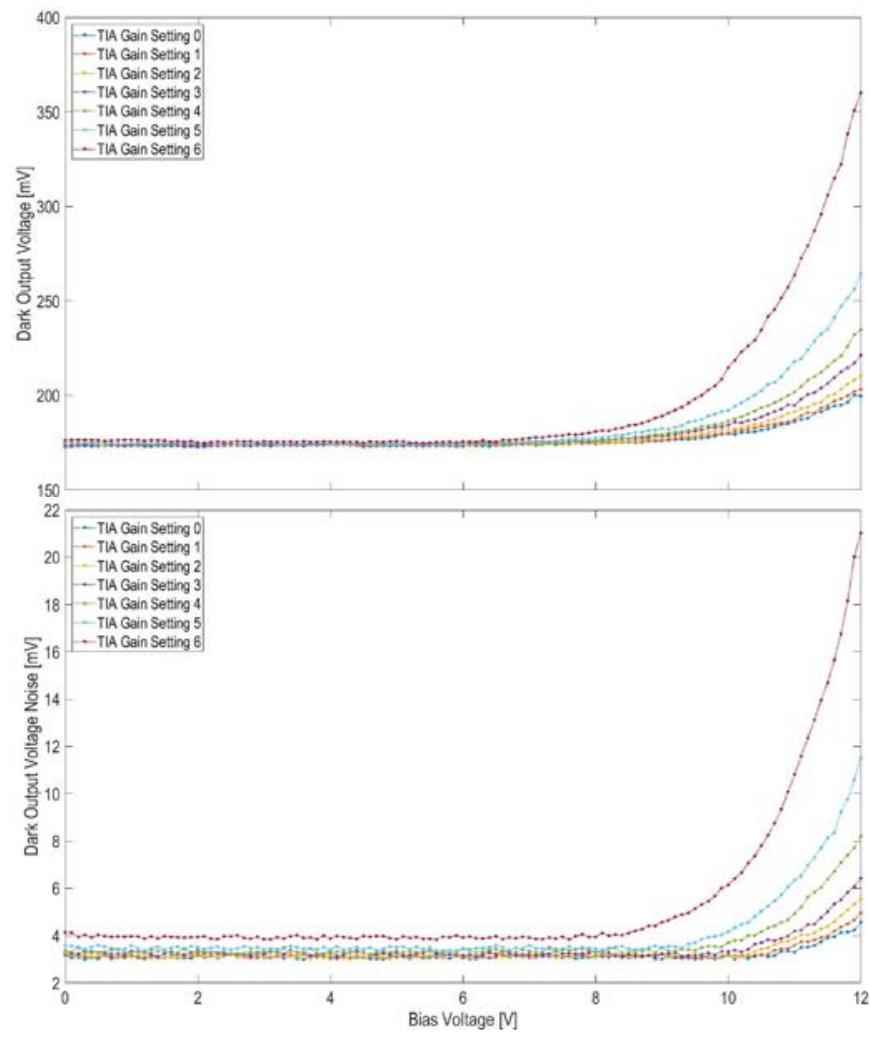

Fig 4. MCT e-APD detection system mean output voltage (top) and output voltage standard deviation or noise (bottom) variations with applied bias at different trans-impedance amplifier gains. Results are obtained in dark condition for the $2 \times 2$ center pixels at a temperature of $77.6 \mathrm{~K}$. Each data point represents the statistics of $30 \mathrm{M}$ Sample record length.

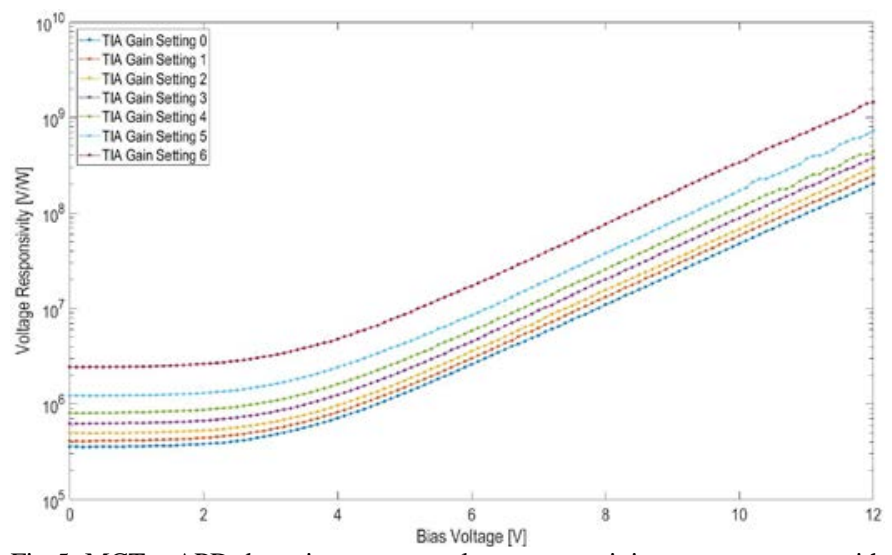

Fig 5. MCT e-APD detection system voltage responsivity measurements with respect to the applied bias voltage at different trans-impedance amplifier gains. Results are obtained for the $2 \times 2$ center pixels at a temperature of $77.6 \mathrm{~K}$. Each data point was obtained by averaging $30 \mathrm{M}$ Sample record length.

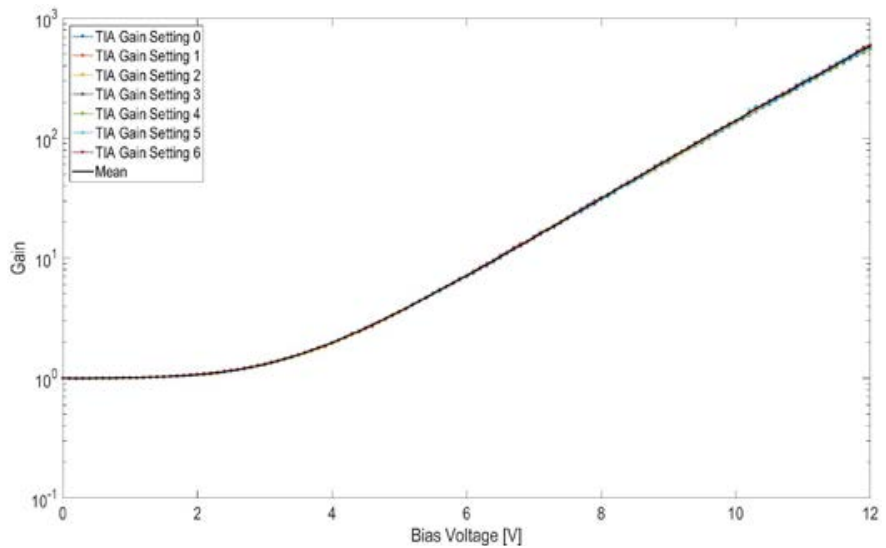

Fig 6. MCT e-APD avalanche multiplication gain measurements with respect to the applied bias voltage at different trans-impedance amplifier gains. Results are obtained by applying Eqs. 1 and 2 to the data of Fig. 5. Solid black curve is the mean value.

The results of Figs. 4 and 5 were applied to calculate the detection system noise-equivalent-power, NEP (in W/ $\mathrm{Hz}^{1 / 2}$ ) and dynamic range as shown in Figs. 7 and 8, respectively. For a fixed operating condition and gain setting, the detection system dynamic range is defined by the minimum and maximum detectable powers, as the lower and upper limits, respectively. The minimum detectable power, $\mathrm{P}_{\min }$ (in $\mathrm{W}$ ), corresponds to the system noise-equivalent-power, NEP (in $\mathrm{W} / \mathrm{Hz}^{1 / 2}$ ), multiplied by the square root of the bandwidth, BW (in $\mathrm{Hz}$ ). This is equivalent to the total measured output voltage noise, $V_{\mathrm{dn}}$, normalized to the voltage responsivity, according to

$$
P_{\min }=\frac{V_{d n}}{\Re_{v}}=N E P \cdot \sqrt{B W}
$$

Through NEP definition, this power results in a unity signal-tonoise ratio (SNR). To insure detection system linearity, the maximum detectable power, $\mathrm{P}_{\max }$ (in $\mathrm{W}$ ), corresponds to the highest radiation that can be applied to the detection system without causing any saturation or clipping in either e-APD detector, electronics or digitizer. This is calculated from 


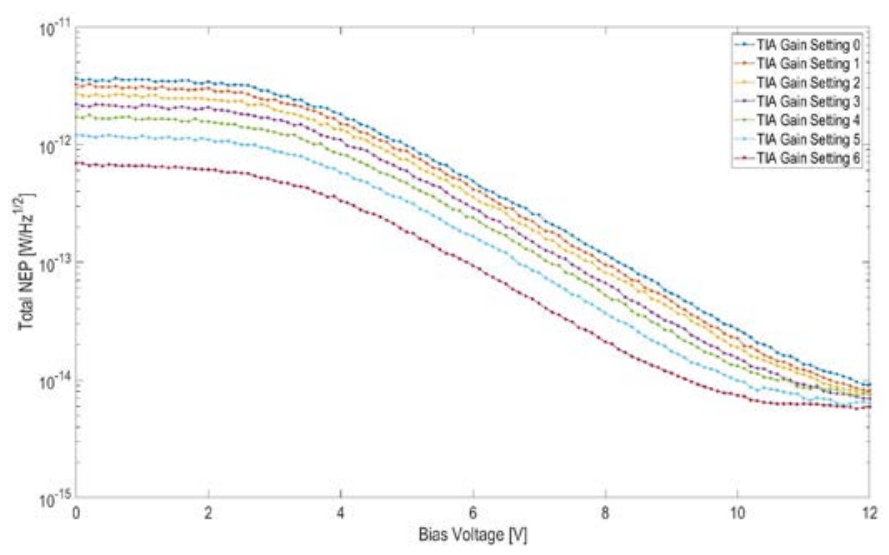

Fig 7. Measured MCT e-APD detection system NEP variation with respect to the applied bias voltage at different trans-impedance amplifier gains. NEP measurements obtained for $2051.04 \mathrm{~nm}$ wavelength at $77.6 \mathrm{~K}$ temperature and include electronic and digitizer noises.

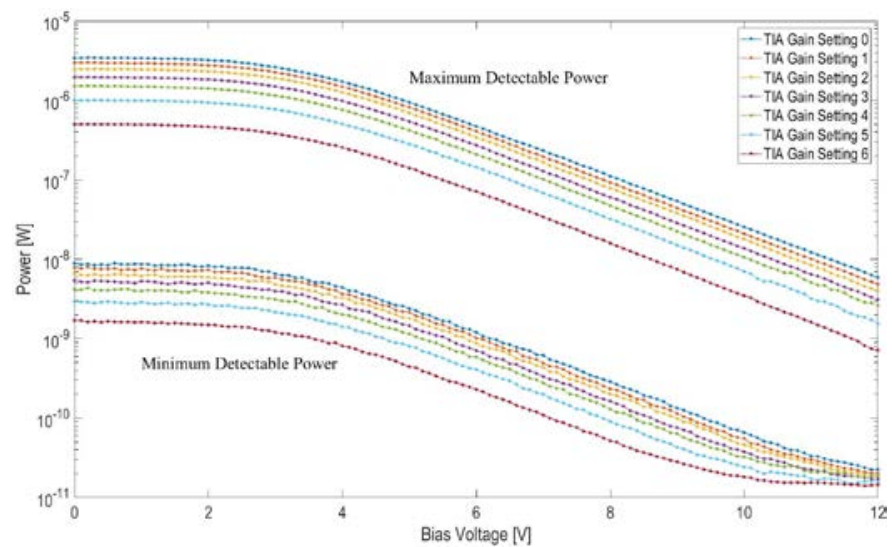

Fig 8. MCT e-APD detection system dynamic range variation with respect to the applied bias voltage at different trans-impedance amplifier gains. At a fixed operating condition, the dynamic range is defined by the minimum and maximum detectable powers. Results are obtained for the $2 \times 2$ center pixels at a temperature of $77.6 \mathrm{~K}$. Saturation of the minimum detectable power is observed at $14 \mathrm{pW}$ independent of the TIA gain.

$P_{\max }=\frac{\left(V_{\max }-V_{d}\right)}{\mathfrak{R}_{v}}$

where $V_{d}$ is the output voltage in dark condition and $V_{\max }$ is the maximum permissible output voltage, set to $1.4 \mathrm{~V}$ in these calculations, which corresponds to the e-APD detection system maximum output limit. Results indicate more than two orders of magnitude input dynamic range, which can be controlled either by the applied bias voltage or the TIA gain. Minimum detectable power limit, or lower saturation, is observed at 14 $\mathrm{pW}$, which is independent of the TIA gain. Considering the system bandwidth of $6 \mathrm{MHz}$, this results in a total detection system NEP of $5.7 \mathrm{fW} / \mathrm{Hz}^{1 / 2}$ equivalent to $1.4 \mathrm{fW} / \mathrm{Hz}^{1 / 2}$ average NEP per pixel.

\section{CONCLUSIONS}

High quality IR detectors are required to enhance the performance of lidar instruments. Detectors based on MCT exhibit high performance within a wide spectral range. Singlecharge-carrier MCT e-APD's are a breakthrough in lidar detection technology as demonstrated through the 1.6- $\mu \mathrm{m}$ technology at GSFC. LaRC has been focusing on several 2- $\mu \mathrm{m}$ lidar transmitter technologies targeting Earth science applications. Combining the $2-\mu \mathrm{m}$ transmitter with this new detector in a single instrument will result in a lidar with enabling technologies that meet space requirements. In coordination with ESTO, GSFC integrated a state-of-the-art lidar detection system using this newly developed e-APD. This system was characterized for 2- $\mu$ m lidar applications at LaRC. Characterization experiments were focused on evaluating the whole detection system, including an advanced digitizer, by measuring dark current, gain and responsivity variations with bias voltage. Quantum efficiency and input dynamic range including NEP and maximum detectable power, were calculated from these results. Operating the detection system using four pixels at $77.6 \mathrm{~K}, 12 \mathrm{~V}$ bias and at the highest TIA gain setting resulted in e-APD gain of 587.5, current responsivity of $615.8 \mathrm{~A} / \mathrm{W}$ and voltage responsivity of 1.45 GV/W. Minimum detectable power of $14 \mathrm{pW}$ was obtained independent of the TIA gain setting, which results in a total detection system NEP of $5.7 \mathrm{fW} / \mathrm{Hz}^{1 / 2}$ equivalent to 1.4 $\mathrm{fW} / \mathrm{Hz}^{1 / 2}$ average NEP per pixel. Work is in progress to integrate and validate this detection system using the newly developed triple-pulse IPDA lidar for simultaneous and independent atmospheric measurements of water vapor and carbon dioxide.

\section{ACKNOWLEDGMENT}

This work was funded and supported by NASA's Earth Science Technology Office. The authors acknowledge the support of NASA Goddard Space Flight Center.

\section{REFERENCES}

[1] Antonio Rogalski, Infrared Detectors, Second Edition, CRC Press, Taylor and Francis Group, 2011.

[2] Antonio Rogalski, "Progress in focal plane array technologies", Progress in Quantum Electronics, 36, 342-473, 2012.

[3] J. Beck, T. Welch, P. Mitra, K. Reiff, X. Sun, and J. Abshire, “A highly sensitive multi-element HgCdTe e-APD detector for IPDA lidar applications”, Journal of Electronic Materials, 43(8), 2970-2977 (2014).

[4] X. Sun, J. Abshire, and J. Beck, "HgCdTe e-APD detector arrays with single photon sensitivity for space lidar applications”, Proc. SPIE, 9114, 91140K, 2014.

[5] U. Singh, B. Walsh, J. Yu, M. Petros, M. Kavaya, T. Refaat, and N. Barnes, "Twenty years of Tm:Ho:YLF and LuLiF laser development for global wind and carbon dioxide active remote sensing”, Optical Materials Express, 5, 827-837, 2015.

[6] T. Refaat, U. Singh, J. Yu, M. Petros, S. Ismail, M. Kavaya, and K. Davis, "Evaluation of an airborne triple-pulsed $2 \mu \mathrm{m}$ IPDA lidar for simultaneous and independent atmospheric water vapor and carbon dioxide measurements,” Applied Optics, 54, 1387-1398, 2015.

[7] U. Singh, M. Petros, T. Refaat, C. Antill, R. Remus and J. Yu, “Airborne lidar for simultaneous measurement of column $\mathrm{CO}_{2}$ and water vapor in the atmosphere", Proc. of SPIE, 10006,1000602, 2016. 